\title{
Etude des relations entre la section du bois d'aubier et la masse foliaire chez le Douglas (Pseudotsuga menziesii Mirb. Franco)
}

\author{
A. GRANIER \\ I.N.R.A., Station de Sylviculture et de Production \\ Centre de Recherches forestières de Nancy, \\ Champenoux, F 54280 Seichamps
}

\begin{abstract}
Résumé
Une étude, entreprise sur Douglas (Pseudorsuga menziesii Mirb. Franco) a permis de mettre en relation la masse foliaire (en matière sèche) et la section de bois d'aubier d'un arbre à un niveau de référence. La relation trouvée, qui est linéaire, est identique pour des plantations de l'Est de la France à celle obtenue dans l'Ouest des Etats-Unis. Cette relation est considérée ici comme traduisant un équilibre biologique spécifique : elle ne dépend ni de l'âge ni de la densité de plantation, ni probablement du site.

Toutefois, des mesures portant cette fois sur des arbres provenant de parcelles ayant été éclaircies il y a six ans, montrent que leur masse foliaire est très supérieure à celle des non éclaircis, par rapport à leur section de bois d'aubier.

Des conséquences en sont tirées du point de vue des modifications du fonctionnement physiologique consécutives à une éclaircie, ainsi qu'au niveau sylvicole.
\end{abstract}

\section{1. - Introduction}

L'importance du houppier des arbres forestiers comme facteur explicatif de leur production a été étudiée depuis quelques années grâce au développement de modèles de fonctionnement et de croissance des arbres forestiers.

Des travaux récents ont fait apparaître que la masse photosynthétique d'un arbre, en équilibre avec son milieu, est fortement corrélée avec la section conductrice efficace du point de vue du flux transpiratoire : le bois d'aubier (GRIER \& Waring, 1974 ; SNell \& Brown, 1978). Il s'agit là d'un équilibre fonctionnel intéressant à deux niveaux :

- biologique, car on peut penser que cet équilibre correspond à un optimum de fonctionnement physiologique. Du point de vue hydrique, le bois d'aubier est le lieu où passe la totalité du flux transpiratoire, mais aussi où s'opèrent des phénomènes d'échanges réversibles d'eau : stockage et restitution. L'importance de la quantité d'eau stockée et disponible dans le bois d'aubier $(10$ à $20 \mathrm{~mm}$ dans des 
peuplements de résineux âgés) provoque un intérêt croissant pour l'étude de ces phénomènes (JARVIS, 1975 ; WARING \& RUNNING, 1978 ; WARING et al., 1979);

- dendrométrique, la relation masse foliaire - section de laubier pouvant fournir un moyen rapide de connaître avec une bonne précision la masse foliaire d"un arbre en peuplement.

Il nous a paru intéressant d'étudier les facteúrs de variation de cet équilibre, ainsi que ses conséquences écophysiologiques et forestières. Nous avons entrepris ce travail dans le cadre d'un programme de recherche sur les bases écophysiologiques de la croissance du douglas. Cette essence, pour laquelle nous disposons de repères bibliographiques abondants, possède un important avenir forestier en France.

\section{2. - Matériel et méthodes}

\section{1. - Le site}

L'étude a été menée en forêt domaniale d'Amance (250 $\mathrm{m}$ d'altitude) dans deux peuplements expérimentaux équiennes de douglas $(*)$. Ces peuplements sont installés sur un sol brun à pseudogley développé sur un substrat imperméable d'argiles liasiques. Leurs principales caractéristiques sont données sur le tableau 1.

Huit arbres ont été prélevés dans le premier dispositif (Arboretum d'Amance) de la façon suivante : un arbre de bordure, deux arbres de circonférence moyenne (CG), et cinq choisis entre la moyenne plus deux écart-types $(C G+2 \sigma)$ et la moyenne moins deux écart-types $(C G-2 \sigma)$. Pour les huit arbres du second dispositif (Carré Latin), trois d'entre cux proviennent d'un plateau non écairci, BI du tableau 1, à 2000 tiges/ha (un arbre de circonférence moyenne CG, un autre de circonférence $\mathrm{CG}+\sigma$, le troisième de circonférence $\mathrm{CG}-\sigma)$; trois autres arbres ont été choisis de la même façon dans un plateau non éclairci, DI, à 1000 tiges/ha. Les deux derniers sont des arbres «moyens» provenant des plateaux éclaircis B II et D II.

Les mesures ont porté sur les différents organes de la partie aérienne.

\section{2. - Mesures au niveau du tronc}

Des rondelles ont été prélevées à plusieurs niveaux de référence dans le tronc : souche, $0,50 \mathrm{~m}, 0,80 \mathrm{~m}, 1,30 \mathrm{~m}$, base de la couronne vivante (BCV), ainsi qu'à des hauteurs intermédiaires : trois rondelles entre $1,30 \mathrm{~m}$ et la base de la couronne, et une rondelle dans chaque entre-nœuds dans la couronne vivante. Ces rondelles ont été colorées au moyen d'une solution de bleu de bromophénol (Kutscha \& SACHs, 1962) qui teinte le bois d'aubier, dont la section, décalquée, est ensuite planimétrée.

(*) Un de ces deux peuplements, une plantation en carré latin, a servi de base à une étude dendrométrique complète (MESSANT, 1980); les mesures de biomasse foliaire nous ont été communiquées par l'auteur. 


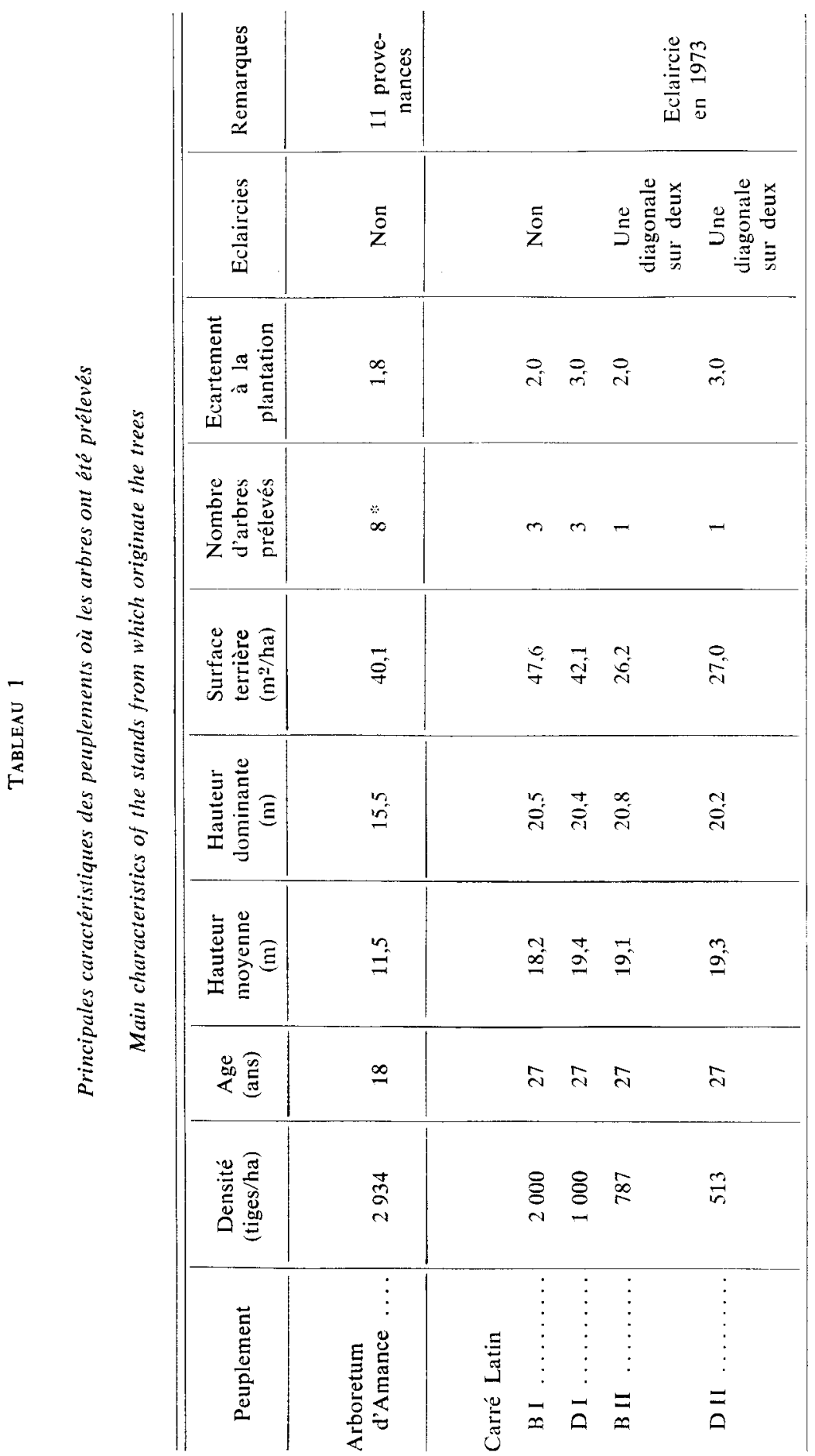




\section{3. - Branches et feuilles}

Sur chaque arbre, toutes les branches vivantes des différents verticilles ont été prélevées et pesées (poids frais). Le poids frais, le poids sec total (après $48 \mathrm{~h}$ à $80^{\circ} \mathrm{C}$ ), le poids sec des aiguilles et de la matière ligneuse ont été mesurés sur une branche échantillon de chaque verticille. La section transversale de chaque branche a été déterminée à $5 \mathrm{~cm}$ de son niveau d'insertion sur la tige.

Enfin, un arbre moyen du peuplement «Arboretum d'Amance» a été choisi pour étudier la répartition des surfaces foliaires. Sur chaque verticille a été prélevé un échantillon des aiguilles des différentes années. Leur surface a été mesurée après projection sur une feuille de papier photographique.

\section{3. - Résultats}

3.1. - Liaison entre la masse foliaire et la section de l'aubier à $1,30 \mathrm{~m}$

La figure 1 reporte pour l'ensemble des arbres étudiés la relation entre la section de l'aubier à $1,30 \mathrm{~m}$ (SA en $\mathrm{cm}^{2}$ ) et la masse foliaire (MF en $\mathrm{kg}$ de poids $\mathrm{sec}$ ).

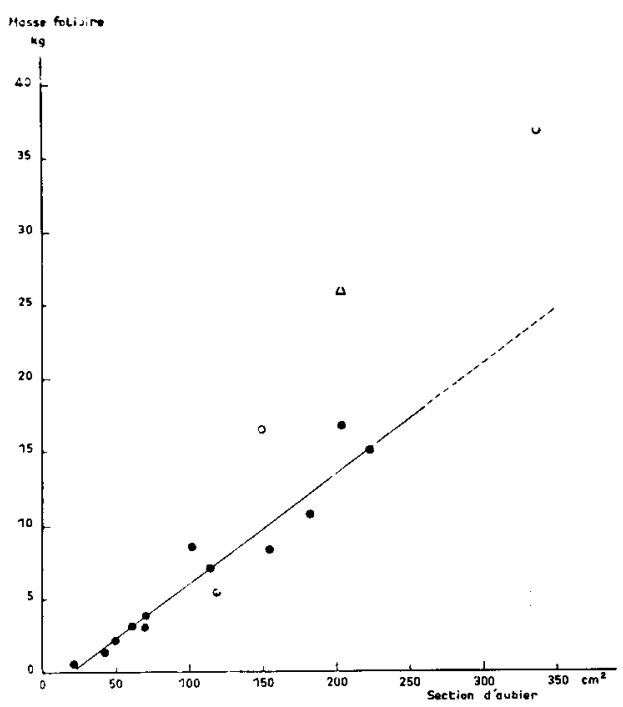

FIG. 1

Relation entre la masse foliaire et la section d'aubier à $1,30 \mathrm{~m}$

Relationship between foliar mass and sapwood cross-sectional area at d.b.h.

- Arbres de placeaux témoins Trees from unthinned plots

Arbres de placeaux éclaircis Trees from thinned plots

$\triangle$ Arbre de bordure Border tree 
Un ajustement linéaire donne la relation :

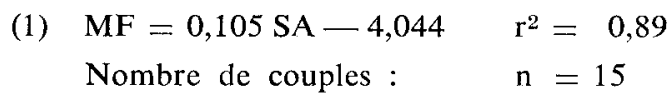

cette relation étant calculée sur tous les arbres, sauf celui de bordure.

L'ajustement de la masse foliaire en fonction de la surface terrière (ST en $\mathrm{cm}^{2}$ ) conduit à la relation :

(2) $\mathrm{MF}=0,054 \mathrm{ST}-2,668 \quad \mathrm{r}^{2}=0,74$

$\mathrm{n}=15$

La section du bois d'aubier à $1,30 \mathrm{~m}$ apparaît donc comme une meilleure variable explicative de la masse foliaire que la section totale (surface terrière).

L'examen de la figure 1 montre que trois arbres sortent du nuage de points; le premier est un arbre de bordure de peuplement, et les deux autres proviennent des placeaux B II à D II où a été pratiquée une éclaircie systématique (une diagonale sur deux) six ans auparavant. Tous les autres arbres proviennent de placeaux n'ayant jamais été éclaircis. Si provisoirement nous éliminons ces arbres, la régression devient :

$$
\begin{array}{ll}
\mathrm{MF}=0,074 \mathrm{SA}-1,365 & \mathrm{r}^{2}=0,92 \\
& \mathrm{n}=14
\end{array}
$$

Le coefficient de corrélation se trouve alors évidemment amélioré. Il est intéressant de constater que cette relation est identique à celle obtenue par GRIER \& WARING (1974) sur la même essence, pour une large gamme d'âges (20 à 130 ans), donc de section d'aubier (jusqu'à plus de $2000 \mathrm{~cm}^{2}$ ) : $\mathrm{MF}=0,074 \mathrm{SA}-1,44$.

\section{2. - Section de l'aubier à différentes hauteurs dans l'arbre}

La décroissance régulière de la section du bois d'aubier avec la hauteur dans l'arbre se fait de façon linéaire, si l'on ne tient pas compte de la mesure au niveau de la souche. Les relations obtenues sont du type :

(4) $\mathrm{SAi}=\mathrm{aHi}+\mathrm{b}$

$\mathrm{SAi}=$ section d'aubier à la hauteur $\mathrm{Hi}$

Dans tous les cas $r^{2}>0,90$, pour $n=11$ à 13 , et les coefficients a et $b$ dépendent chacun de la masse foliaire de l'arbre.

La figure 2 schématise cette décroissance caractéristique.

Si l'on considère le degré de liaison entre la masse foliaire et la section du bois d'aubier à différentes hauteurs, il semble que la base de la couronne vivante soit un meilleur niveau de référence que la hauteur $1,30 \mathrm{~m}$. En effet on obtient, pour les arbres des dispositifs non éclaircis, y compris un arbre de bordure :

(5) $\quad \mathrm{MF}=0,115 \mathrm{SA}-1,721 \quad \mathrm{r}^{2}=0,98$ 


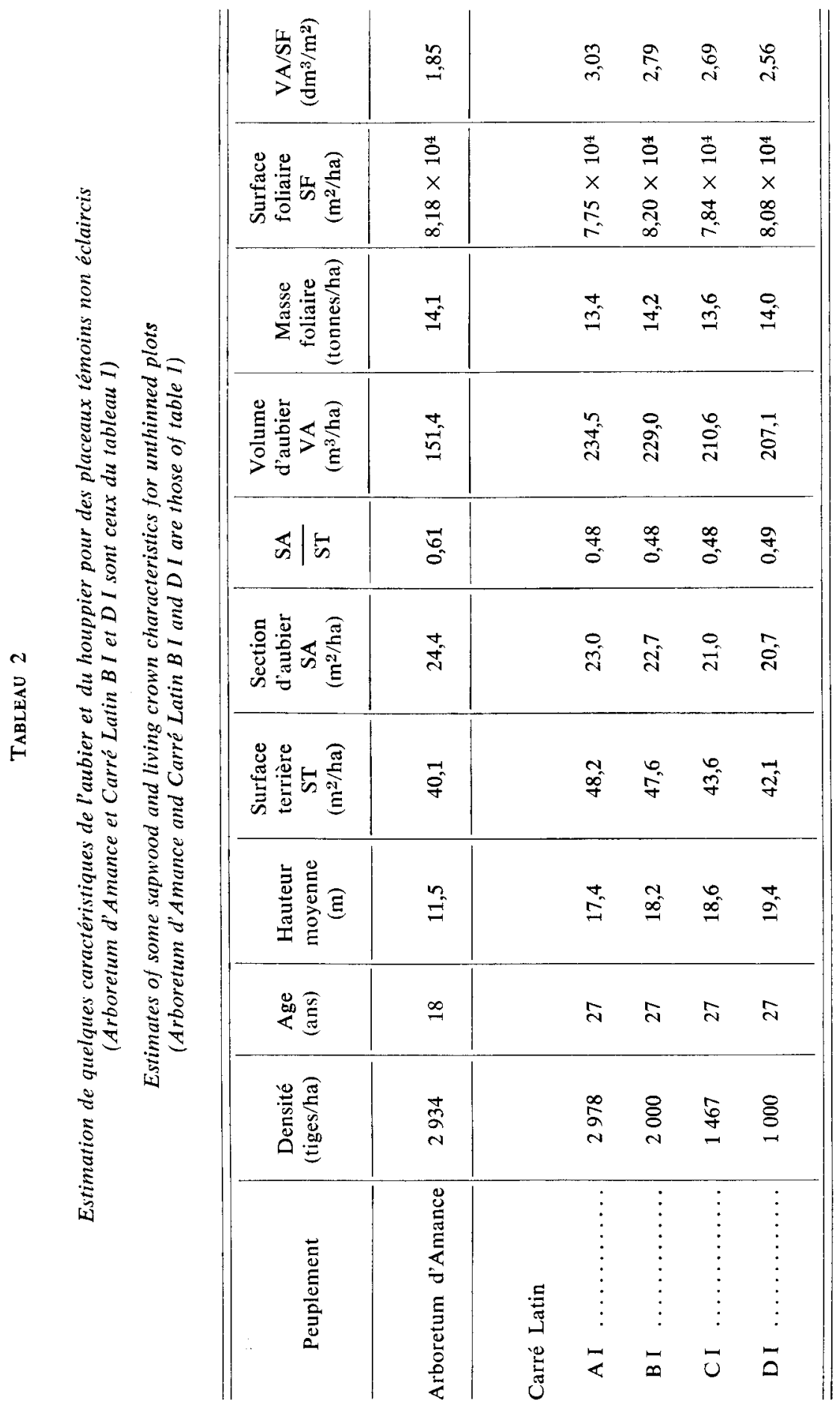




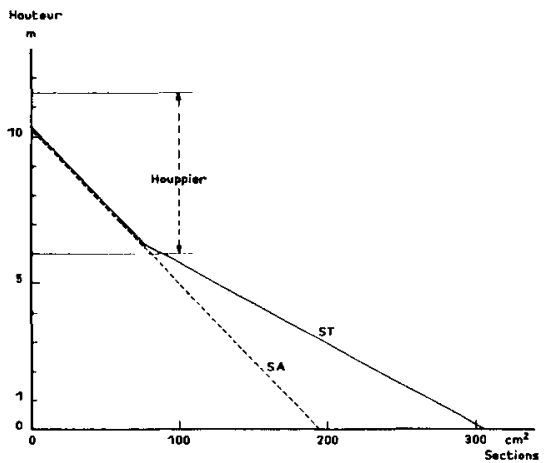

Fig. 2

Variation de la section d'aubier $S A$ ef de la section fotale $S T$ avec la hauteur dans le tronc

Variation of sapwood (SA) and total (ST) cross sectional area with height in the trunk

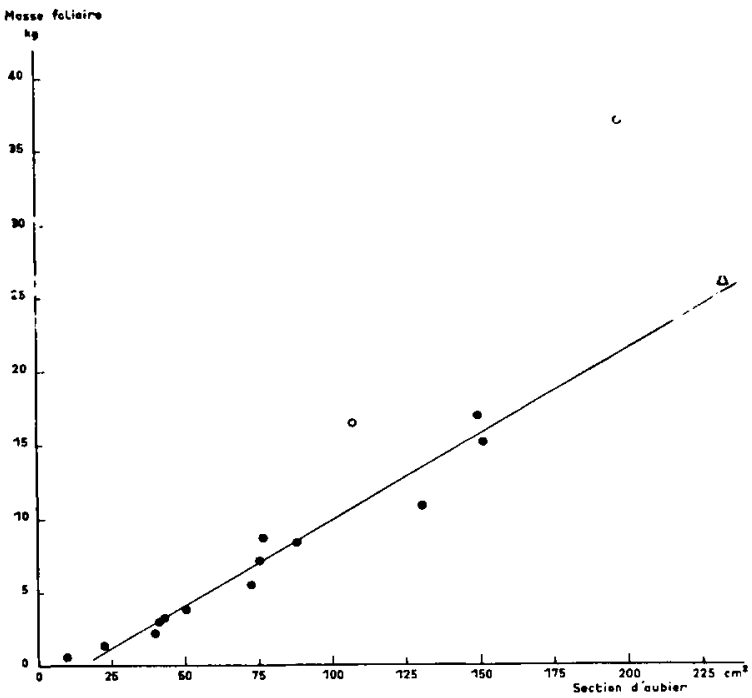

Fig. 3

Relation entre la masse foliaire et la section d'aubier à la base de la couronne vivante Relationship between foliar mass and sapwood cross-sectional area at the base of the bole

- Arbres de placeaux témoins

Trees from unthinned plots

Arbres de placeaux éclaircis

Trees from thinned plots

$\triangle$ Arbre de bordure

Border tree 
Nous constatons aussi (figure 3) que l'arbre de bordure ne sort pas de la relation déterminée par les arbres de peuplement. Par contre, comme nous l'avions remarqué au $\$ 2.1$., les deux arbres provenant de placeaux éclaircis s'écartent de la relation (5), de façon très significative.

Enfin, il existe aussi une relation linéaire entre la masse foliaire et la section conductrice au niveau des branches; la relation (6) est obtenue sur 52 branches :

$$
\mathrm{MFB}=0,135 \mathrm{SAB}-0,105 \quad \mathrm{r}^{2}=0,92
$$

\section{3. - Estimations dans les placeaux non éclaircis}

Le tableau 2 donne les estimations respectives de la section d'aubier et de son volume, de la masse foliaire et de sa surface, valeurs rapportées à l'hectare. Ces estimations ont été faites pour des placeaux témoins non éclaircis; les arbres de l'échantillon ont été prélevés dans trois d'entre eux : Arboretum d'Amance, et Carré Latin (B I et D I).

La surface foliaire, mesurée sur un arbre moyen, est de $5,79 \mathrm{~m}^{2}$ (une face d'aiguilles seulement) pour $1 \mathrm{~kg}$ de poids $\mathrm{sec}$ d'aiguilles.

Deux autres placeaux du Carré Latin y ont été introduits (A I et CI).

Il est à remarquer que face à l'homogénéité des sections d'aubier, masses et surfaces foliaires, les volumes d'aubier rapportés à l'hectare présentent d'importantes différences entre l'Arboretum d'Amance et les quatre blocs du Carré Latin. Si l'on rapporte le volume d'aubier à l'unité de masse foliaire, on constate que $1 \mathrm{~m}^{2} \mathrm{de}$ surface foliaire est supporté par $1,85 \mathrm{dm}^{3}$ d'aubier pour l'Arboretum d'Amance et de 2,56 à $3,03 \mathrm{dm}^{3}$ pour les placeaux témoins du Carré Latin.

\section{4. - Discussion - Conclusion}

La mesure de la section conductrice de sève brute à $1,30 \mathrm{~m}$ a montré une étroite liaison linéaire avec la masse foliaire, et donc la surface transpirante. Cette relation apparaît, chez le Douglas, indépendante de l'âge et de la densité de plantation, tout au moins pour des peuplements non éclaircis.

La surface foliaire alimentée par une unité de surface conductrice est donc constante : $1 \mathrm{~cm}^{2}$ de bois d'aubier à $1,30 \mathrm{~m}$ alimente environ $60 \mathrm{~g}$ soit $0,35 \mathrm{~m}^{2}$ de feuilles.

L'équilibre entre surfaces transpirante et conductrice est encore plus affirmé au niveau de la base de la couronne vivante. Mais dans le houppier la section de l'aubier et la section totale à une certaine hauteur ont le même poids pour expliquer la masse foliaire au-dessus de cette hauteur. La prise en compte de la section d'aubier plutôt que la section totale comme variable explicative de la masse foliaire n'améliore la régression que dans la partie du tronc située au-dessous de la couronne vivante. 
Le cas des arbres provenant de placeaux éclaircis en 1973 est intéressant à considérer. Les deux arbres ont une masse foliaire plus importante que les autres par rapport à leur section d'aubier. On sait que l'éclaircie provoque une augmentation de la masse des houppiers, comme l'ont montré Aussenac \& Boulangeat, 1980, par des mesures d’interception des précipitations dans des placettes de Douglas éclaircies et témoins.

Toutefois, Helms, 1964 n’a pas montré d'accroissement durable du taux de photosynthèse chez le Douglas après éclaircie, et le gain de production photosynthétique n'est dû qu'à un gain de masse foliaire. On peut alors faire l'hypothèse qu'il y a à la fois accroissement de la longévité de la base des couronnes vivantes qui ne «remontent» pas, couplé à une plus forte production d’aiguilles dans les zones inférieures du houppier anciennement en contact. Nous pensons que le déséquilibre ainsi créé par laugmentation de la masse foliaire tendra progressivement à disparaître par accroissement de la section conductrice; le problème est alors de connaître la cinétique de ce phénomène.

L'augmentation de la section du bois d'aubier ne peut logiquement se faire que par un accroissement de la surface terrière (la formation du bois de cour est un phénomène irréversible); on peut d'ailleurs considérer que l'accroissement maximum de l'aubier s'effectue pour une extension nulle du bois de cœur.

Le phénomène de retour à cet équilibre masse foliaire-section d'aubier a pu être montré dans le cas inverse : un élagage artificiel provoque une diminution progressive et significative du bois d'aubier chez le peuplier (SACHSSE, 1965).

La sylviculture vise à atteindre un optimum entre la production et la qualité des produits, par l'intensité et la fréquence des éclaircies.

Or, l'éclaircie provoque une modification des deux équilibres fonctionnels : la section conductrice et le volume d'eau échangeable, rapportés à l'unité de masse foliaire, diminuent. De plus, les houppiers sont placés, après éclaircie, dans une situation microclimatique où la demande transpiratoire est plus élevée; les arbres sont donc mis en position défavorable face aux stress hydriques. Il convient donc que l'intensité de l'éclaircie soit suffisamment forte pour qu'il y ait un gain sensible de masse foliaire (donc de productivité), mais sans trop accroître la sensibilité des arbres vis-à-vis des contraintes hydriques. On peut alors penser que la fréquence des éclaircies ne doit pas être supérieure au temps mis par les arbres en peuplement pour revenir à leur état d’équilibre.

Reçu pour publication en décembre 1980.

\section{Summary}

Study of relationships between sapwood cross sectional area and foliar mass of Douglas-fir (Pseudotsuga menziesii Mirb. Franco)

A study on Douglas-fir (Pseudotsuga menziesii Mirb. Franco) have shown a relationship between the foliar mass (dry weight) and the cross sectional sapwood area of a tree at d.b.h. This linear relationship obtained in plantations of eastern France, is the same as that found in western U.S.A. Since this relationship is independant of the age, the density and probably the site, it implies a specific biological equilibrium. Nevertheless, in plots which have been 
thinned six years ago, the foliar mass of the trees is much greater than that of unthinned plots with respect to their sapwood cross section.

In discussion, changes in physiological behaviour following the thinning, and sylvicultural consequences are examinated.

\section{Références bibliographiques}

Aussenac G., et Boulangeat C., 1980. Interception des précipitations et évapotranspiration réelle dans des peuplements de feuillus (Fagus silvatica L.) et de résineux (Pseudotsuga menziesii [Mirb.] Franco). Ann. Sci. for., 37 (2), 91-107.

Grier C.C. et Waring R.H., 1974. Conifer foliage mass related to sapwood area. For. Sci. Note, 20 (3), 205-206.

HeLms J.A., 1964. Apparent photosynthesis of Douglas-fir in relation to silvicultural treatment. For. Sci., 10 (4), 432-442.

JARVIS P.G., 1975. Water transfer in plants. In : Heat and mass transfer in the biosphere. I. - Transfer processes in plant environment, D.A. de Vries et N.K. van Alfen (Editors). 1974 Seminar of the International Centre for Heat and Mass Transfer, Dubrovnik. Scripta Book Co., Washington D.C., 369-394.

KuTSCHA N.P. et SACHS I.B., 1962. Color tests for differentiating heartwood and sapwood in certain softwood trees species. U.S.D.A. For. Ser. Resour. Bull. F.P.L.-2246, 17 p. Forest Prod. Lab., Madison, WI.

Messant D., 1980. Contribution à la sylviculture du douglas. Eléments de sa production. Mémoire E.N.I.T.E.F., 64 p. Publication Station de Sylviculture et de Production du C.N.R.F. $\mathrm{n}^{\circ} 80 / 03$.

RunNing S.W., 1980. Relating plant capacitance to the water relations of Pinus Contorta. For. Ecol. and Manage., 2 (4), 237-252.

SACHSSE H., 1965. Investigation of the influence of pruning on the formation of coloured heartwood and tension wood in some Poplar species. Traduction C.S.I.R.O. $\mathbf{n}^{\circ} 7929$, 16 p., 1966. Traduit de Holz als Roh -und Werkstoff, 23 (11), 425-434.

SNELL K.J.A. et Brown J.K., 1978. Comparison of tree biomass estimators DBH and Sapwood area. For. Sci., 24 (4), 455-457.

WARING R.H. et RunNing S.W., 1978. Sapwood water storage : its contribution to transpiration and effect upon water conductance through the stems of old-growth Douglas-fir. Plant Cell Environ., 1, 131-140.

Waring R.H., Whitehead D. et Jarvis P.G., 1979. The contribution of stored water to transpiration in Scots pine. Plant Cell Environ., 2 (4), 309-317. 